\title{
A Consumer-Oriented Car Style Evaluation System Based on Fuzzy Mathematics and Neural Network
}

\author{
Wenhui Hou ${ }^{1}$, Caiwen $\mathrm{Niu}^{2 *}$ \\ ${ }^{1}$ Institute or University or Company, Department of Mechanical Engineering, Tangshan Polytechnic \\ College, Tangshan 063299, China \\ ${ }^{2}$ Department of Automation Engineering, Tangshan Polytechnic College, \\ Tangshan 063299, China \\ *Email: niucaiwen0305@126.com
}

Received: January 31, 2021. Revised: July 28, 2021. Accepted: August 13, 2021. Published: August 24, 2021.

\begin{abstract}
As an important link in product development, car style evaluation could ensure the quality of car style design, making the design more efficient, laying the foundation for production planners, production managers, and investment decision-makers in automobile manufacturing. The consumercentered evaluation should accurately reflect the psychological cognition and subjective feelings of consumers. However, the current studies have not provided a unified evaluation standard, nor fully utilized the massive data on the evaluations made by consumers. Considering in advantages of fuzzy mathematics and neural network in processing massive data on consumer evaluations, this paper designs a consumer-oriented car style evaluation system based on these two techniques. Firstly, a scientific evaluation index system was designed for consumeroriented car style evaluation, the index scores were classified into different levels, and a judgment matrix was constructed for indices on each layer and subject to consistency check. Next, absolute weights were assigned to alternatives, and the corresponding fuzzy membership functions were determined, producing a fuzzy comprehensive evaluation (FCE) model based on analytic hierarchy process (AHP) (AHP-FCE model) for car style evaluation. Furthermore, car styles were categorized by appearance structure, and the car style samples were parametrized for evaluation. Finally, particle swarm optimization (PSO) was improved, and then combined with backpropagation neural network (BPNN) into a classification model for consumeroriented car style evaluation. The proposed consumer-oriented car style evaluation model was proved effective and superior through experiments. The results offer a reference for the application of the model in other evaluation scenarios.
\end{abstract}

Keywords-Fuzzy mathematics, neural network, car style, consumer-oriented evaluation

\section{INTRODUCTION}

$\mathrm{C}$ ar style evaluation aims to identify the optimal car style solution by assessing and comparing the values of different car style designs during the design phase [1-2]. As an important link in product development, car style evaluation could ensure the quality of car style design, making the design more efficient, laying the foundation for production planners, production managers, and investment decision-makers in automobile manufacturing [3-4].

Traditionally, the feasible car style designs are selected by expert scoring organized by carmakers [5-9]. With simple indices, this approach is too subjective to satisfy the carmakers' need for consumer-oriented precision evaluation of car style. The sales of cars, which are not cheap for common households, is greatly affected by the individual preferences of consumers. With strong subjectively, high randomness, and simple indices, the traditional evaluation methods cannot meet the growing demand for consumer-oriented car style evaluation. This calls for mathematical evaluation methods and tools, capable of analyzing, reasoning, and calculating the quantitative targets in car style designs.

Due to the complexity of car style evaluation, the enterprises hope to judge the qualitative evaluation parameters under the guidance of consumer preference, apart from the assessment of quantitative targets [10-13]. Car body is the focus of car style design with cultural and artistic features. Scholars like Lin and Papadopoulos [17] and Montemanni et al. [18] held that car style design originates from the aesthetic needs of designers and consumers, and starts from visual perception [14-18].

Singh and Tando [19] designed experiments for car style evaluation, collected electroencephalogram (EEG) signals from the subjects, and carried out feature extraction and feature analysis, revealing the significant disparity between the brain electrical activities corresponding to different evaluation results [20-22]. To cater to consumers' personal preference to car appearance, Garces et al. [23] selected several perceptual 
imagery words that characterize the consumers' subjective feeling of car appearance, organized them into an evaluation index system for car appearance, conducted a Kansei engineering survey based on the eight style feature lines of car appearance, and performed a fuzzy comprehensive evaluation (FCE) experiment on a production instance [24-26]. Sun [27] noticed that the optimization efficiency and direction are affected by the inconsistency in evaluation results of multiple subjects, constructed a multi-subject car style evaluation and analysis system through detailed demand analysis, and realized the interactive design of multiple functional modules in car evaluation system, such as multi-subject decision-making, component selection, as well as result generation and display. From the dimensions of human and materials, Jing [28] summarized the influencing factors of car style, set up an evaluation index system for car style in the design phase, and established an evaluation model based on the neural network, thereby ensuring the effectiveness of weight coefficients, and reducing the human impacts in coefficient setting.

The rapid progress of Internet information technology makes it possible to survey the consumers in the phase of car design. The existing studies have shown that, the suggestions and opinions of consumers, being purchasers and users of cars, can affect the decisions on car style design to a certain extent. But there is not yet a unified stanard. Therefore, it is necessary to develop a consumer-oriented evaluation method that accurately reflect the psychological cognition and subjective feelings of consumers.

Considering in advantages of fuzzy mathematics and neural network in processing massive data on consumer evaluations, this paper designs a consumer-oriented car style evaluation system based on these two techniques. The remainder of this paper is organized as follows: Section 2 establishes an FCE model based on analytic hierarchy process (AHP) (AHP-FCE model) for car style evaluation through multiple steps, namely, building an evaluation index system for consumer-oriented car style evaluation, classification of index scores, construction and consistency check of the judgment matrix for indices on each layer, assigning absolute weights to alternatives, and determining fuzzy membership functions; Section 3 categorizes car styles by appearance structure, and parameterizes the car style samples to be evaluated; Section 4 improves the particle swarm optimization (PSO), and combines it with backpropagation neural network (BPNN), creating a classification model for consumer-oriented car style evaluation; Section 5 verifies the effectiveness and superiority of the proposed model for car style evaluation; Section 6 summarizes the findings of this research, laying the basis for applying our model to other evaluation scenarios.

\section{COnStRuction OF EVAluation IndeX System}

Consumer-oriented car style evaluation faces several problems: multiple influencing factors, complex hierarchy, and difficulty in index quantification. To overcome these problems, this paper combines the AHP and fuzzy mathematics to build up an AHP-FEC model for consumer-oriented car style evaluation, divides the evaluation indices into different levels, determines membership functions, and assigns weights to alternatives.

Before building the AHP-FEC model, the authors sorted out the literature of the evaluation index system for car style evaluation, and constructed an evaluation index system with consumer willingness at the core, from the perspectives of availability, artistry, and technicality. As shown in Table 1, the proposed evaluation index system contains five primary indices (design creativity, design scientificity, design form, design color, and design texture) and fourteen secondary indices.

To objectively describe the consumer-oriented evaluation of the practicality, aesthetics, and economics of car style, this paper designs five evaluation levels: A (poor), B (general), C (good), D (high-quality), and $\mathrm{E}$ (excellent). The relative importance between indices was determined against the 1-9 scale of the AHP. Table 2 presents the judgement matrix for the criteria layer. The consistency ratio of the matrix was smaller than 0.10 , passing the consistency test. Thus, the matrix is feasible for evaluation.

Table 1. Evaluation index system for consumer-oriented car style evaluation.

\begin{tabular}{|c|c|c|}
\hline Goal layer & Criteria layer & Alternative layer \\
\hline \multirow{14}{*}{$\begin{array}{c}\text { Consumer- } \\
\text { oriented car style } \\
\text { evaluation } M D\end{array}$} & \multirow{3}{*}{$\begin{array}{c}\text { Design } \\
\text { creativity } M D_{1}\end{array}$} & New thinking $M D_{11}$ \\
\hline & & New concept $M D_{12}$ \\
\hline & & New form $M D_{13}$ \\
\hline & \multirow{3}{*}{$\begin{array}{c}\text { Design } \\
\text { scientificity } \\
M D_{2}\end{array}$} & $\begin{array}{l}\text { Functional completeness } \\
\qquad M D_{21}\end{array}$ \\
\hline & & $\begin{array}{l}\text { Human-machine } \\
\text { coordination } M D_{22}\end{array}$ \\
\hline & & Safety and reliability $M D_{23}$ \\
\hline & \multirow{3}{*}{$\begin{array}{l}\text { Design form } \\
\qquad M D_{3}\end{array}$} & $\begin{array}{c}\text { Streamline smoothness } \\
M D_{31}\end{array}$ \\
\hline & & $\begin{array}{c}\text { Proportional harmony } \\
M D_{32}\end{array}$ \\
\hline & & Function-style unity $M D_{33}$ \\
\hline & \multirow{3}{*}{$\begin{array}{l}\text { Design color } \\
\qquad M D_{4}\end{array}$} & $\begin{array}{c}\text { Function-color } \\
\text { coordination } M D_{41}\end{array}$ \\
\hline & & $\begin{array}{l}\text { Environment-tone } \\
\text { coordination } M D_{42}\end{array}$ \\
\hline & & $\begin{array}{l}\text { Primary color-secondary } \\
\text { color coordination } M D_{43}\end{array}$ \\
\hline & \multirow{2}{*}{$\begin{array}{c}\text { Design texture } \\
M D_{5}\end{array}$} & Visual texture $M D_{51}$ \\
\hline & & Tactile texture $M D_{52}$ \\
\hline
\end{tabular}

Table 2. Judgement matrix of the criteria layer.

\begin{tabular}{|c|c|c|c|c|c|}
\hline $\mathrm{MD}$ & $\mathrm{MD}_{1}$ & $\mathrm{MD}_{2}$ & $\mathrm{MD}_{3}$ & $\mathrm{MD}_{4}$ & $\mathrm{MD}_{5}$ \\
\hline $\mathrm{MD}_{1}$ & 1 & 0.25 & 0.5 & 0.33 & 0.5 \\
\hline $\mathrm{MD}_{2}$ & 2 & 1 & 2 & 2 & 3 \\
\hline $\mathrm{MD}_{3}$ & 3 & 0.33 & 1 & 0.33 & 2 \\
\hline $\mathrm{MD}_{4}$ & 4 & 0.5 & 2 & 1 & 2 \\
\hline $\mathrm{MD}_{5}$ & 2 & 0.33 & 3 & 2 & 1 \\
\hline
\end{tabular}

Table 3. Absolute weights of alternatives.

\begin{tabular}{|c|c|c|c|c|c|c|}
\hline \multirow{3}{*}{$\begin{array}{c}A-B \\
\text { sortin } \\
\mathrm{g}\end{array}$} & \multicolumn{5}{|c|}{ T-A sorting } & Absolut \\
\cline { 2 - 6 } & $M D_{1}$ & $M D_{2}$ & $M D_{3}$ & $M D_{4}$ & $M D_{5}$ & $\begin{array}{c}\text { value } \\
\text { in }\end{array}$ \\
& $\begin{array}{c}0.074 \\
1\end{array}$ & $\begin{array}{c}0.145 \\
2\end{array}$ & $\begin{array}{c}0.243 \\
2\end{array}$ & $\begin{array}{c}0.278 \\
3\end{array}$ & $\begin{array}{c}0.259 \\
2\end{array}$ & $\begin{array}{c}\text { overall } \\
\text { sorting }\end{array}$ \\
\hline
\end{tabular}


INTERNATIONAL JOURNAL OF CIRCUITS, SYSTEMS AND SIGNAL PROCESSING

\begin{tabular}{|c|c|c|c|c|c|c|}
\hline$M D_{11}$ & $\begin{array}{c}0.241 \\
5\end{array}$ & & & & & 0.01789 \\
\hline$M D_{12}$ & $\begin{array}{c}0.463 \\
1\end{array}$ & & & & & 0.03431 \\
\hline$M D_{13}$ & $\begin{array}{c}0.295 \\
4\end{array}$ & & & & & 0.02188 \\
\hline$M D_{21}$ & & $\begin{array}{c}0.116 \\
8\end{array}$ & & & & 0.01695 \\
\hline$M D_{22}$ & & $\begin{array}{c}0.352 \\
8\end{array}$ & & & & 0.05122 \\
\hline$M D_{23}$ & & $\begin{array}{c}0.530 \\
4\end{array}$ & & & & 0.07701 \\
\hline$M D_{31}$ & & & $\begin{array}{c}0.342 \\
7\end{array}$ & & & 0.08334 \\
\hline$M D_{32}$ & & & $\begin{array}{c}0.536 \\
6\end{array}$ & & & 0.13050 \\
\hline$M D_{33}$ & & & $\begin{array}{c}0.120 \\
7\end{array}$ & & & 0.02935 \\
\hline$M D_{41}$ & & & & $\begin{array}{c}0.528 \\
6\end{array}$ & & 0.14710 \\
\hline$M D_{42}$ & & & & $\begin{array}{c}0.123 \\
6\end{array}$ & & 0.03439 \\
\hline$M D_{43}$ & & & & $\begin{array}{c}0.347 \\
8\end{array}$ & & 0.09679 \\
\hline$M D_{51}$ & & & & & $\begin{array}{c}0.421 \\
4\end{array}$ & 0.10922 \\
\hline$M D_{52}$ & & & & & $\begin{array}{c}0.578 \\
6\end{array}$ & 0.14997 \\
\hline
\end{tabular}

There are certain correlations between the indices on different layers. Based on the AHP-FEC model, the absolute weight of each alternative could be derived from the mutual influence between indies, which is characterized by the judgement matrix. The absolute weights of alternatives are recorded in Table 3.

As quantitative indices, the alternatives are not suitable for mathematical processing. To solve the problem, the scores of the alternatives were divided into five levels $[1,0.8],[0.8,0.6]$, $[0.6,0.4],[0.4,0.2]$, and $[0.2,0]$, which in turn corresponds to strongly good, slightly good, medium, slightly poor, and strongly poor. The membership functions corresponding to the five levels can be expressed as:

$$
\begin{gathered}
\mu_{1}(x)=\left\{\begin{array}{lc}
0 & x \leq 0.1 \\
3-9 x & 0.1<x \leq 0.2 \\
0 & x>0
\end{array}\right. \\
\mu_{2}(x)=\left\{\begin{array}{lc}
0 & x \leq 0.1 \\
9 x-2 & 0.1<x \leq 0.2 \\
1 & 0.2<x \leq 0.3 \\
5-9 x & 0.3<x \leq 0.4 \\
0 & x>0.4
\end{array}\right. \\
\mu_{3}(x)=\left\{\begin{array}{lc}
0 & x \leq 0.3 \\
9 x-4 & 0.3<x \leq 0.4 \\
1 & 0.4<x \leq 0.5 \\
7-9 x & 0.5<x \leq 0.6 \\
0 & x>0.6
\end{array}\right.
\end{gathered}
$$

$$
\begin{gathered}
\mu_{4}(x)=\left\{\begin{array}{lc}
0 & x \leq 0.5 \\
10 x-5 & 0.5<x \leq 0.6 \\
1 & 0.6<x \leq 0.7 \\
8-10 x & 0.7<x \leq 0.8 \\
0 & x>0.8
\end{array}\right. \\
\mu_{5}(x)=\left\{\begin{array}{lc}
0 & x \leq 0.7 \\
10 x-8 & 0.7<x \leq 0.8 \\
1 & x>0.8
\end{array}\right.
\end{gathered}
$$

Based on the above evaluation index system, this paper establishes the AHP-FCE model for consumer-oriented car style evaluation by classifying index scores, building and testing the consistency of the judgment matrix for indices on each layer, assigning absolute weights to alternatives, and determining fuzzy membership functions. The quantified evaluation results were expressed as a set $Q L=\{A, B, C, D, E\}$.

The evaluation process can be divided into two phases, namely, first FCE, and second FCE. During the first FCE, five weight vectors $W_{2-i}$ were constructed for the alternatives, and the fuzzy matrix $F M=\left\{F_{1}, F_{2}, F_{3}, F_{4}, F_{5}\right\}$ and fuzzy vectors $V_{i}$ $=W_{2-i} F_{i}$ corresponding to $Q L$ were generated by computing the membership function.

During the second FCE, a five-row five-column fuzzy matrix $C_{j}$ was established based on $V_{i}$. Let $W_{1-j}$ be the weight vector of criteria. Then, the second FCE results were obtained by computing $C=W_{1-j} V_{i}$.

The validity $\eta$ of the maximum membership principle in consumer-oriented car style evaluation can be calculated by:

$$
\eta=\frac{\alpha^{\prime}}{\beta^{\prime}}=\frac{N \alpha-1}{2 \beta(N-1)}
$$

where, $N$ is the number of alternatives; $\beta$ and $\gamma$ are the proportions of the alternative with the largest weight and the second-largest weight in the sum of all weights. Table 4 presents the correspondence between the value range of validity and validity of maximum membership principle.

Table 4. Correspondence between the value range of validity and validity of maximum membership principle.

\begin{tabular}{|c|c|}
\hline $\begin{array}{c}\text { Value range of validity } \\
\eta\end{array}$ & $\begin{array}{c}\text { Validity of maximum membership } \\
\text { principle }\end{array}$ \\
\hline 0 & Invalid \\
\hline$(0,0.5)$ & Poorly valid \\
\hline$[0.5,1)$ & Moderately valid \\
\hline$[1+\infty)$ & Strongly valid \\
\hline$+\infty$ & Extremely strongly valid \\
\hline
\end{tabular}

\section{SAMPLE PARAMETERIZATION}

The car style was categorized by appearance structure to improve the classification accuracy of the scores on the alternatives. The car style designers' cognition of aesthetics, practicality and economy can be expressed through their car style designs. The design details could be sensed by consumers when they actually control and operate the car.

After observing the image samples of car style, the authors performed morphological analysis to extract elements from 
different car styles, including bumper style $E_{1}$, wing plate style $E_{2}$, engine cover style $E_{3}$, door style $E_{4}$, headlight style $E_{5}$, car body feature lines $E_{6}$, radiator grid style $E_{7}$, sliding roof style $E_{8}$, and door frame style $E_{9}$. The nine style elements were divided into 27 categories by form, material, and color, to describe the features of the samples to be evaluated.

To parameterize the samples, the elements belonging to form features were subject to the curve control method. Firstly, the key points were determined on the feature lines at each part of the sample. Then, the coordinates of the key points were adopted as the basis for parametrizing the samples to be evaluated.

Take the car body feature lines, which carry the largest amount of sample information, for example. Let $K_{1}, K_{2}, K_{3}, K_{4}$, $K_{5}, K_{6}$, and $K_{7}$ be the seven key points on one side; $R_{1}$ and $R_{2}$ be the front and rear round corner arc radii, respectively; $L_{1}, L_{2}$, and $L_{3}$ be front, middle, and rear widths, respectively; $L_{4}, L_{5}$, and $L_{6}$ be the front, middle, and rear lengths, respectively (Figure 1). AutoCAD was employed to determine the coordinates of the key points. For both front and side views, the horizontal-vertical coordinate system was constructed with the center of the car style image as the origin. Table 5 provides the values of form features for the car body feature lines in a car style. The eigenvalues of other form features are similar to these values.

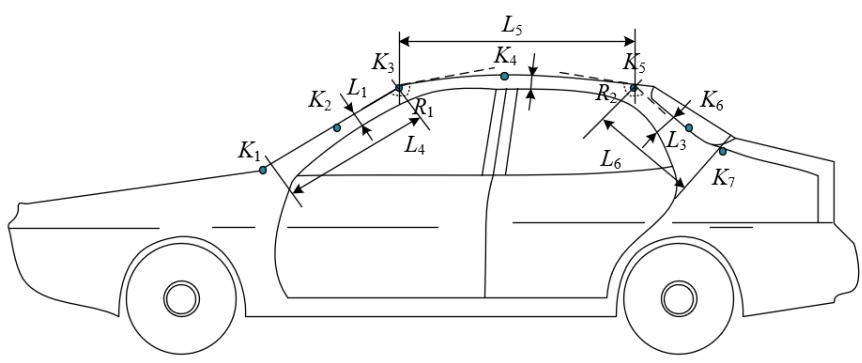

Fig. 1 Key points and form features of car style feature lines

Table 5. Values of form features for car body feature lines.

\begin{tabular}{|c|c|c|c|c|}
\hline Key point & \multicolumn{2}{|c|}{ Coordinates } & Form feature & Eigenvalue \\
\hline$K_{1}$ & $a_{1}=-47.3$ & $b_{1}=5.2$ & $R_{1}$ & 169.3 \\
\hline$K_{2}$ & $a_{2}=-35.3$ & $b_{2}=14.7$ & $R_{2}$ & 161.1 \\
\hline$K_{3}$ & $a_{3}=21,6$ & $b_{3}=22.1$ & $L_{1}$ & 12.3 \\
\hline$K_{4}$ & $a_{4}=0$ & $b_{4}=21.4$ & $L_{2}$ & 5.2 \\
\hline$K_{5}$ & $a_{5}=19.4$ & $b_{5}=20.4$ & $L_{3}$ & 9.8 \\
\hline$K_{6}$ & $a_{6}=29.4$ & $b_{6}=13.9$ & $L_{4}$ & 56.8 \\
\hline$K_{7}$ & $a_{7}=36.3$ & $b_{7}=4.7$ & $L_{5}$ & 130.2 \\
\hline & & & $L_{6}$ & 50.1 \\
\hline
\end{tabular}

Based on the eigenvalues of form features, the degree of aesthetics for car style design was analyzed through clustering, providing a reference for the scoring of alternatives. The analysis results were also divided into five levels: $[1,0.8]$, $[0.8,0.6],[0.6,0.4],[0.4,0.2]$, and $[0.2,0]$, which in turn corresponds to strongly good, slightly good, medium, slightly poor, and strongly poor.

Style texture and style color are both qualitative parameters. The former was divided into strong texture (1) and weak texture $(0)$, and the latter into coordinated with surrounding components (1) and uncoordinated with surrounding components (0). Table 6 provides the values of the features for a car style sample.

Table 6. Values of the features for a car style sample.

\begin{tabular}{|c|c|c|c|c|c|c|c|c|c|}
\hline Featur & \multicolumn{10}{|c|}{ Style element } \\
\cline { 2 - 11 } & $E_{1}$ & $E_{2}$ & $E_{3}$ & $E_{4}$ & $E_{5}$ & $E_{6}$ & $E_{7}$ & $E_{8}$ & $E_{9}$ \\
\hline Form & $\begin{array}{c}0.6 \\
7\end{array}$ & $\begin{array}{c}0.8 \\
5\end{array}$ & $\begin{array}{c}0.4 \\
8\end{array}$ & $\begin{array}{c}0.9 \\
3\end{array}$ & $\begin{array}{c}0.8 \\
7\end{array}$ & $\begin{array}{c}0.2 \\
7\end{array}$ & $\begin{array}{c}0.4 \\
6\end{array}$ & $\begin{array}{c}0.6 \\
8\end{array}$ & $\begin{array}{c}0.9 \\
0\end{array}$ \\
\hline $\begin{array}{c}\text { Textur } \\
\mathrm{e}\end{array}$ & 1 & 1 & 0 & 1 & 0 & 0 & 1 & 1 & 0 \\
\hline Color & 1 & 1 & 1 & 1 & 1 & 0 & 1 & 1 & 1 \\
\hline
\end{tabular}

For each target car style sample, the authors established a set of scores for alternatives and a dataset of sample features, providing the data basis for building the classification model based on neural network.

\section{Construction of Classification Model}

Figure 2 presents the proposed classification model for consumer-oriented car style evaluation. For car style evaluation, BPNN is good at self-learning, adaptation, generalization, nonlinear mapping, and fault tolerance. However, this neural network faces a number of defects: susceptibility to local minimum, slow convergence, nonuniform structure, and sample dependance. To prevent these defects, this paper improves the PSO and combines it with BPNN to build up a classification model for consumer-oriented car style evaluation.

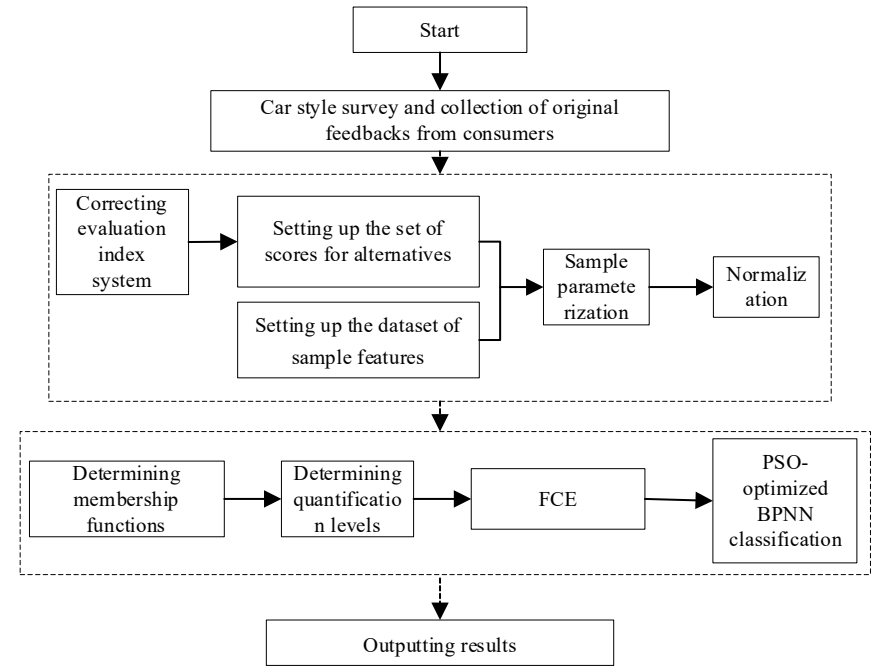

Fig. 2 Structure of our classification model for consumer-oriented car style evaluation 


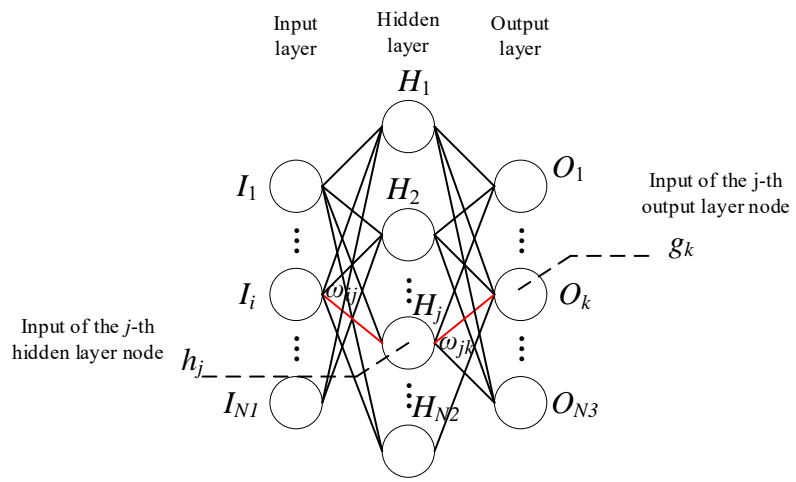

Fig. 3 Structure of our BPNN

The training of BPNN is an iterative update of network weights and thresholds. Figure 3 shows the structure of the proposed BPNN. Let $S=\left\{\left(I_{1}, O_{1}\right),\left(I_{2}, O_{2}\right), \ldots,\left(I_{N 1}, O_{N 1}\right)\right\}, I \in \mathbb{R}^{N 1}, O$ $\in \mathbb{R}^{N 3}$ be the set of target car style samples; $\varepsilon_{2-j}$ and $\varepsilon_{3-k}$ be the thresholds of the $\mathrm{j}$-th hidden layer node, and $\mathrm{k}$-th output layer node, respectively; $h_{j}$ and $g_{k}$ be the inputs of the $\mathrm{j}$-th hidden layer node, and k-th output layer node, respectively. Taking sigmoid as the activation function, the BPNN output can be expressed as:

$$
\hat{O}_{k}=\operatorname{Sigmoid}\left(g_{k}-\varepsilon_{3-k}\right)
$$

The mean squared error (MSE) between actual and expected outputs can be computed by:

$$
M S E=\frac{1}{2} \sum_{k=1}^{N_{3}}\left(\hat{O}_{k}-O_{k}\right)^{2}
$$

The weights and thresholds of the network can be updated by:

$$
\phi=\phi+\Delta \phi
$$

BPNN adjusts its parameters by gradient descent. Thus, the parameter variation can be computed by:

$$
\Delta \phi_{j k}=-\alpha \cdot \frac{\partial M S E}{\partial \phi_{j k}}=\alpha \cdot \frac{\partial M S E}{\partial \hat{O}_{k}} \cdot \frac{\partial \hat{O}_{k}}{\partial g_{k}} \cdot \frac{\partial g_{k}}{\partial \phi_{j k}}
$$

The PSO is a bionic optimization algorithm that guides particles to search for the optimal solution in a specific space. Each particle with its velocity and position represents a potential solution. Let $M$ be the swarm size; $V_{i}=\left(v_{i, 1}, v_{i, 2}, \ldots, v_{i, m}\right)$ and $P_{i}=\left(p_{i, 1}, p_{i, 2}, \ldots, p_{i, m}\right), m=1,2, \ldots, M$ be the velocity and position of the $\mathrm{i}$-th particle in the $\mathrm{t}$-th iteration, respectively. Then, the velocity and position of each particle can be iteratively updated by:

$$
\begin{gathered}
V_{i}^{t+1}=\delta V_{i}^{t}+a_{1} \eta_{1}\left(\Psi_{i}^{t}-P_{i}^{t}\right)+a_{2} \eta_{2}\left(\Psi_{G}^{t}-P_{i}^{t}\right) \\
P_{i}^{i+1}=P_{i}^{t}+V_{i}^{t+1}
\end{gathered}
$$

where, $\Psi_{i}^{t}$ and $\Psi_{G}^{t}$ are the individual and global best solutions, respectively; $\delta \in[0,1]$ is the inertia weight; $a_{1}$ and $a_{2}$, both equal 1.5 , are learning factors; $\eta_{1}$ and $\eta_{2}$, both falling in $[0,1]$, are two random numbers.

This paper improves the PSO by swarm division and probabilistic mutation of particles. The fitness of each particle was calculated, and the swarm was divided during the optimization. Let $v$ be a sufficiently small integer. If $v$ satisfies formula (13), $Q^{t}$ is a high-quality particle; otherwise, $Q^{t}{ }_{i}$ is a low-quality particle:

$$
\frac{\text { Fitness }\left(P_{i}^{t}\right)}{\text { Fitness }_{\text {avg }}^{t}} \leq v
$$

where, Fitness ${ }_{\text {avg }}$ is the mean fitness of particles in the t-th generation.

$$
\text { Fitness }_{\text {avg }}^{t}=\frac{1}{M} \sum_{i=1}^{M} \text { Fitness }\left(P_{i}^{t}\right)
$$

The high-quality particles were organized into the highquality swarm $G_{E-u}^{t}$, and the low-quality particles into the lowquality swarm $G_{I-v}^{t}, u=1,2, \ldots, M_{1}, u=1,2, \ldots, M_{1}+M_{2}=M$. Let $v_{s}$ and $v_{e}$ be the initial and final values of $v$, respectively; $t_{M A X}$ be the maximum number of iterations. Then, the swarm can be divided by dynamic adjustment of $v$ :

$$
v=v_{2}-\frac{t_{M A X}-t}{t_{M A X}}\left(v_{2}-v_{1}\right)
$$

During the swarm division, the low-quality swarm contains many particles in the early iterative process, suggesting that the particle diversity can be improved by increasing the number of mutable particles; the low-quality swarm contains fewer particles in the late iterative process, indicating that the potential solutions represented by the particles approach the optimal solution. Let $P_{S}{ }_{S}$ be the position of a particle after mutation. Then, the particles in the low-quality swarm can be mutated by the mutation operator:

$$
P_{S}^{t}=P_{I-l}^{t}+\rho_{v} \sqrt{\sum_{w=1}^{M_{2}}\left(X_{l-w}^{t}-X_{\Psi_{G}^{t}-w}^{t}\right)^{2}} \varphi \circ V_{I-l}^{t}
$$

where, $\phi$ is the direction coefficient in the form of a $1 \times D$ dimensional matrix (every element in the matrix is a random number in $[1,1]$; o is the Hadamard product operation of the matrix.

Formula (16) shows that $P_{S}^{t_{S}}$ depends on the Euclidean distance between the global optimal position and the position of the 1-th particle in the t-th iteration. The mutation probability $\rho_{v}$ can be calculated by:

$$
\rho_{v}=\frac{\text { Fitness }\left(P_{I-l}^{t}\right)}{\sum_{l=1}^{M_{2}} \text { Fitness }\left(P_{I-l}^{t}\right)}
$$

The probabilistic mutation above shows that the inferior particles have a large Euclidean distance from the global optimal solution in the early phase of iterations. This phase features a strong global search ability. When it comes to the middle phase of iterations, the said Euclidean distance becomes small. In this phase, the particles have a strong local search ability. In the late phase of iterations, the distance is minimized, and the particles gradually converge to the global optimal.

The PSO-improved BPNN consists of the following steps:

Step 1. Determine the number of nodes in the input, hidden, and output layers of the $\operatorname{BPNN}\left(N_{1}, N_{2}\right.$, and $\left.N_{3}\right)$; select the activation function for each layer; determine the maximum number of iterations for the network $\mathrm{T}$; initialize the learning rate.

Step 2. Determine swarm size $M$, particle dimensions $U$, maximum and minimum velocities of particles $v_{M A X}$ and $v_{M I N}$, 
and maximum number of iterations $t_{M A X}$; set the initial weight, final weight, and learning factors; initialize the swarm information, as well as the initial velocity and position of each particle. Among them, particle dimensions $U$ can be derived from the number of nodes in the input, hidden, and output layers of the BPNN:

$$
U=N_{1} \times N_{2}+N_{2} \times N_{3}+N_{2}+N_{3}
$$

Step 3. Set the fitness function of the swarm as the MSE function of the BPNN:

$$
\text { Fitness }=\frac{1}{M} \sum_{c=1}^{M} \sum_{i=1}^{N_{3}}\left(\hat{O}_{i}^{c}-O_{i}^{c}\right)^{2}
$$

Step 4. Obtain the individual optimal solution $\Psi_{i}{ }_{i}$ and global optimal solution $\Psi_{G}^{t}$. Divide the swarm into a high-quality swarm $G_{E-u}^{t}$ and a low-quality swarm $G_{I-v}^{t}$. Retain the highquality swarm in the next iteration, and perform particle mutation on the low-quality swarm to generate a child swarm with new positions and velocities.

Step 5. Obtain the individual optimal solution $\Psi^{t}{ }_{i}$ and global optimal solution $\Psi_{G}^{t}$ again. If the swarm fitness is better than the individual optimal solution, retain the velocity and position of the particle; otherwise, update the velocity and position of the particle by formulas (11) and (12).

Step 6. Judge if the current number of iterations is smaller than the maximum number of iterations. If yes, go back to Step 4; otherwise, terminate the iteration, output the optimal solution of the swarm, and initialize the weights and thresholds of the BPNN.

Step 7. Compute the MSE of BPNN output, and update weights and thresholds of network. Judge if the current number of iterations is greater than the maximum number of iterations T. If yes, terminate the training; otherwise, re-calculate the MSE of the output.

\section{Procedure of CONSUMER-ORIENTEd CAR STYle EVALUATION}

Figure 4 presents the workflow of the proposed consumeroriented car style evaluation model. Referring to Figure 4, the common steps of our model can be detailed as follows:

Step 1. Correct the evaluation index system for consumeroriented car style evaluation based on the scores given by experts and designers.

Step 2. Based on the original consumer feedbacks in the car style survey, construct the set of alternative scores and the dataset of sample features.

Step 3. To eliminate the dimensional difference between the scores and feature attribute data, normalize the data before importing them into the model for training, following the guidance of Figure 4. The normalization formula can be expressed as:

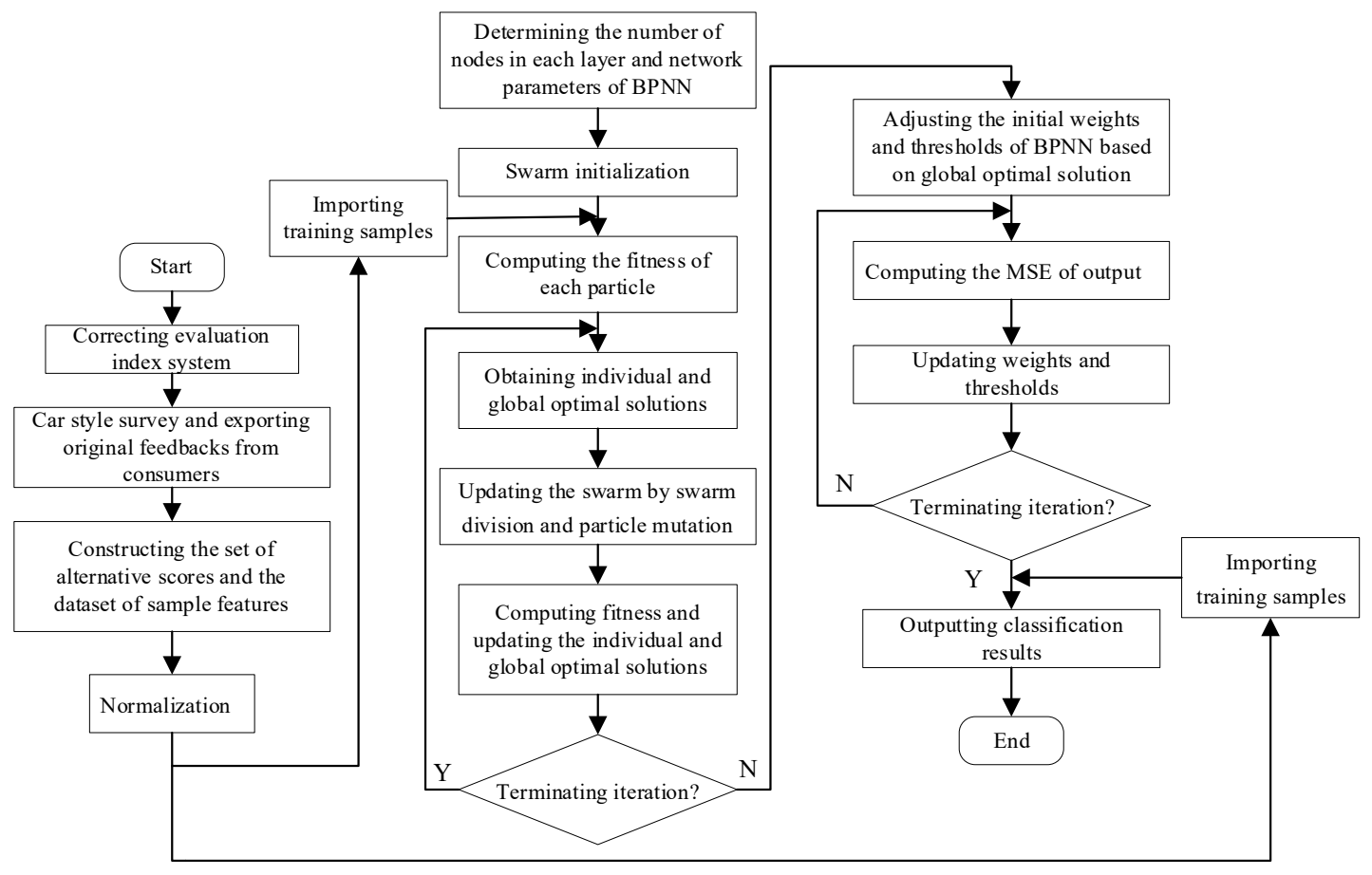

Fig. 4 Workflow of our model

$$
\left\{\begin{array}{l}
x_{i j}=\frac{s_{i j}-\min \left(s_{i j}\right)}{\max \left(s_{i j}-\min \left(s_{i j}\right)\right)} \\
x_{i j}^{\prime}=\frac{\max \left(s_{i j}\right)-s_{i j}}{\max \left(s_{i j}\right)-\min \left(s_{i j}\right)}
\end{array}\right.
$$

where, $x$ and $x^{\prime}$ are positive and negative indices, respectively.

Step 4. Train the BPNN with normalized sample set, using error backpropagation algorithm. As shown in Figure 4, apply the trained BPNN to consumer-oriented car style evaluation, and output the evaluation results.

Step 5. Compare the evaluation results, and provide 
suggestions on how to optimize car styles and incentivize car style design.

\section{EXPERIMENTS AND RESULTS ANALYSIS}

Based on set of alternative scores and the dataset of sample features, the authors established a sample library of car style evaluation, and designed an experiment to verify the scientificity of the proposed evaluation index system and the effectiveness of our model. Figure 5 compares the convergence conditions of the PSO and the improved PSO. Before improvement, the PSO converged to the optimal solution at the 65-th iteration, where the particle fitness was 0.0083 . The improved PSO converged to the optimal solution at the124-th iteration, where the particle fitness was 0 . The result is consistent with the global optimal value of the swarm given by fitness function Ackley, indicating that the improved algorithm can effectively prevent premature convergence to the local minimum.

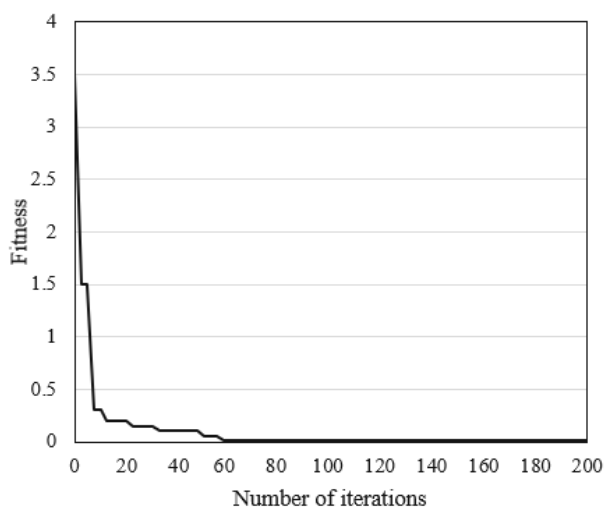

(a) PSO

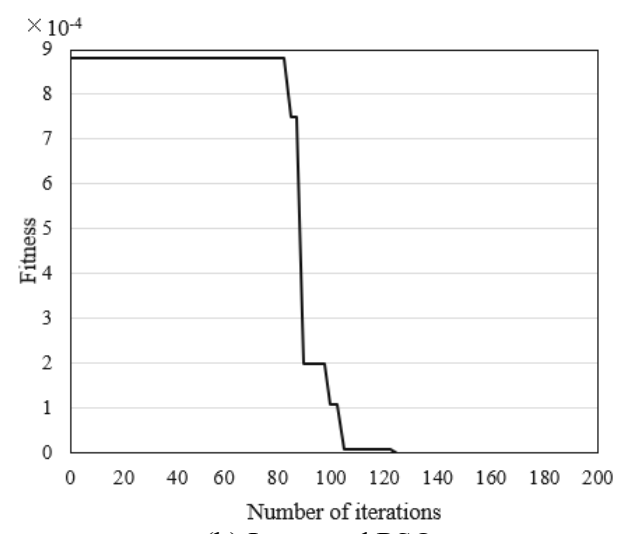

(b) Improved PSO

Fig. 5 Convergence conditions of PSO and improved PSO

Moreover, a contrastive experiment was designed to verify whether sample parameterization could effectively enhance the correctness of the classification of alterative scores. Figure 6 compares the FCE results obtained before and after sample parameterization. After the samples were parametrized, the scores of different car styles fluctuated less significantly, and obeyed a more reasonable and reliable distribution. The more the consumers involved in the evaluation, the more realistic the FCE results. For example, the score of sample 4 dropped from the exaggerated value of 4.97 to 4.89 . Meanwhile, the fewer the consumers involved in the evaluation, the closer the FCE results to the mean score. For example, the score of sample 17 slightly increased from 4.51 to 4.59 .

Further, the proposed BPNN-based classification model was trained and tested on 500 sets of car style evaluation samples. Figure 7 records the losses and accuracies of the BPNN. After 60 iterations, the network error dropped below 0.01 , the training accuracy reached $97.14 \%$, and the test accuracy arrived at $96.33 \%$.

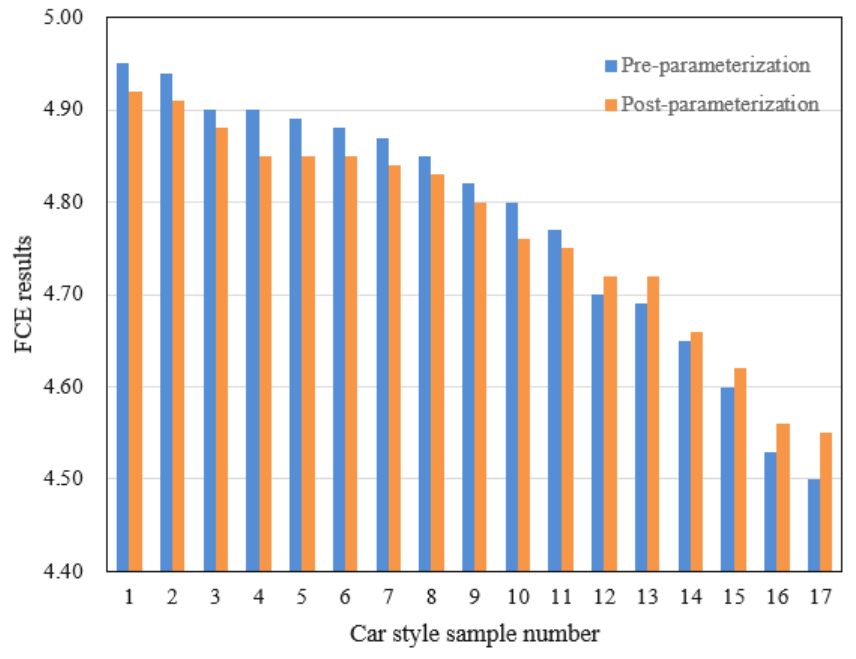

Fig. 6 FCE results before and after sample parameterization

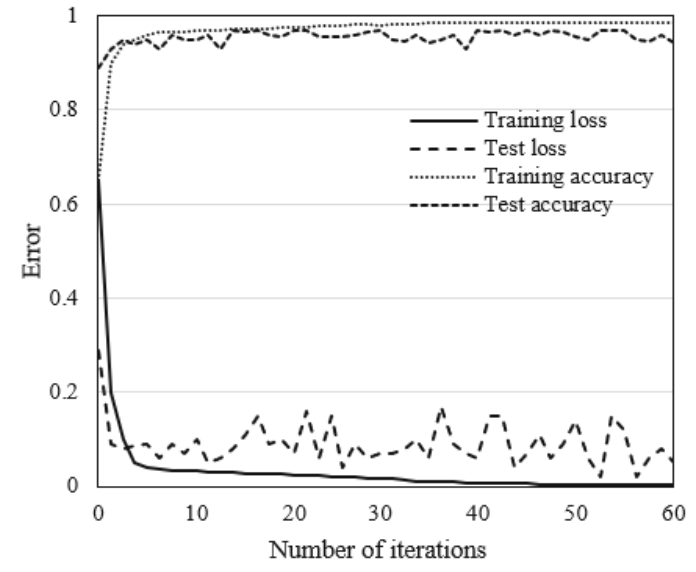

Fig. 7 Losses and accuracies of the BPNN

Based on our classification model, a human-machine interaction system was created for consumer-oriented car style evaluation. The system was constructed under the browser/server (B/S) architecture on Windows XP. The webpages were designed with the aid of Macromedia Dreamweaver. As shown in Figure 8, the system mainly consists of a frontpage to display car styles, a car style evaluation page, and a car style analysis page. The webpages can display the car styles, and allow consumers to evaluate each car style and upload their scores. The uploaded data are used to enrich the data library. The evaluation and analysis pages support the upload of car style images, and consumer-oriented evaluation of image samples. The evaluation results help to improve the design plan or compute consumer satisfaction. 


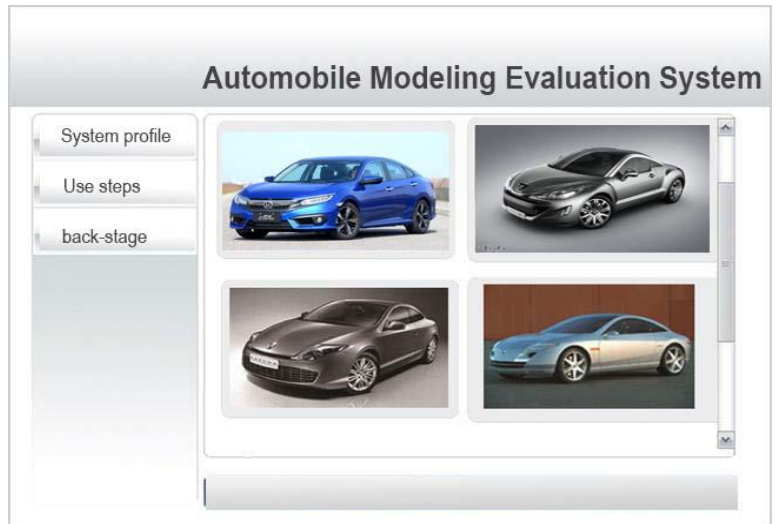

(a)

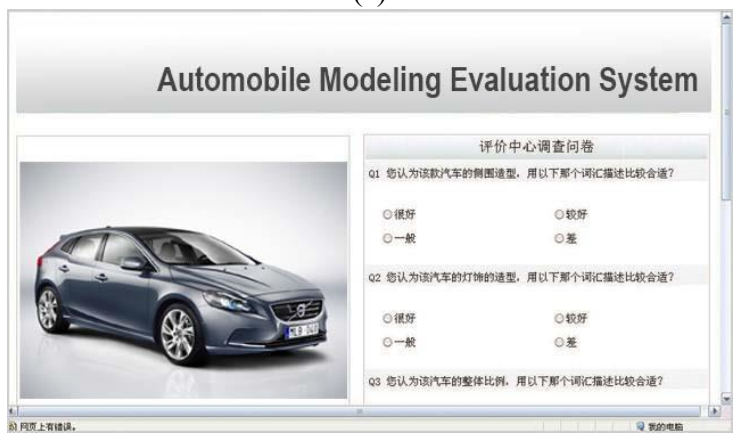

(b)

\section{Automobile Modeling Evaluation System}

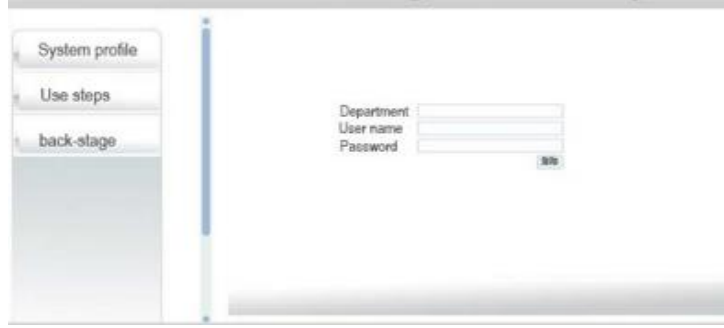

(c)

Fig. 8 Human-machine interaction interface of our classification model

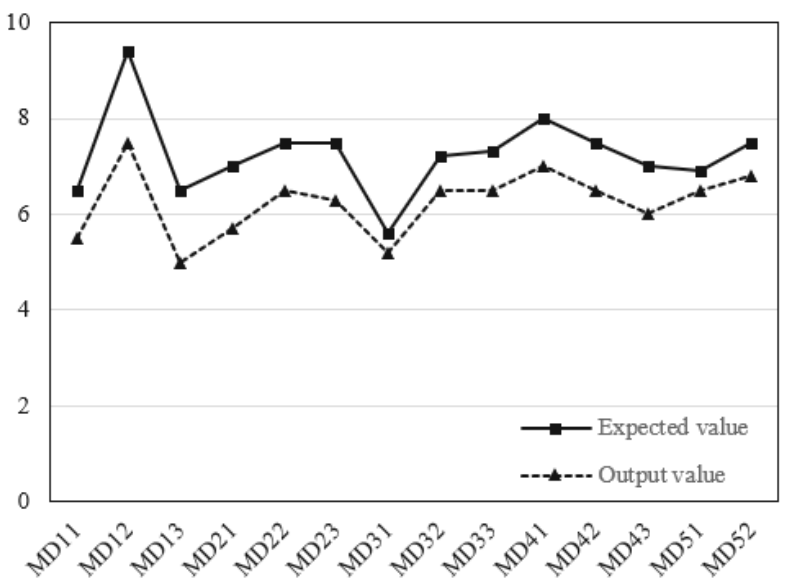

Fig. 9 Comparison between expected value and actual output

Figure 9 compares the expected value with the actual output. The expected values of alternatives changed in a similar trend as the model outputs. The output was slightly below the expected value, due to the neglection of the correlation between multiple indices. To further verify the classification accuracy of our model, Figure 10 compares the classification results before and after PSO improvement. The classification results of the improved PSO were almost identical to the actual values, suggesting the superiority of our algorithm on consumeroriented car style evaluation to the traditional algorithms.

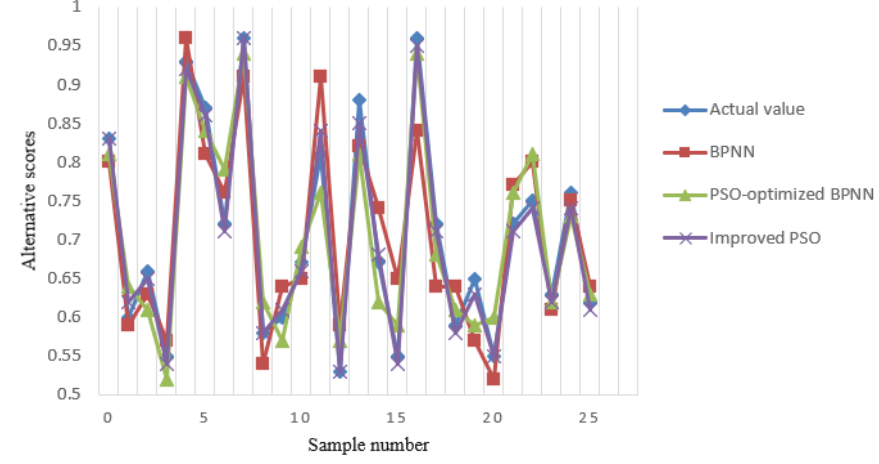

Fig. 10 Comparison of prediction results

\section{CONCLUSIONS}

The consumer-centered evaluation should accurately reflect the psychological cognition and subjective feelings of consumers. However, the current studies have not provided a unified evaluation standard. The fuzzy mathematics and neural network can process massive data more effectively than most other algorithms. For this reason, this paper designs a consumer-oriented car style evaluation system based on fuzzy mathematics and neural network. Firstly, an AHP-FCE model was established for car style evaluation through multiple steps, namely, building an evaluation index system, classification of index scores, construction and consistency check of the judgment matrix for indices on each layer, assigning absolute weights to alternatives, and determining fuzzy membership functions. Next, the car style was classified by appearance structure to parametrize the car style samples to be evaluated. The FCE results before and after the parameterization of some car style samples were compared through experiments. The experimental results show that the parameterization of samples made the evaluation scores of diferent car styles less volatile, and more reasonable and reliable in distribution. Finally, improved PSO was coupled with BPNN to classify the results of consumer-oriented car style evaluation. The human-machine interaction system of the proposed car style evaluation model was developed, and the classification effect of the system was tested through experiments. The exepcted values of our model were compared with the actual outputs. It was found that the exepcted values under each criterion had the same trend as the model outputs. The classification results of our model were almost identical to the actual values, suggesting the superiority of our algorithm on consumer-oriented car style evaluation to the traditional algorithms. The research results provide a complete consumer-oriented evaluation system and classification model for car styles for the readers of the journal, and lay the basis for the application of relevant models in other evaluation systems or evaluation fields. 


\section{References}

[1] H. Q. Liang, X. F. Feng, J. Y. Tang, Q. F. Liu, “Confidence reliability evaluation model of dual constant-stress accelerated life test for Weibull distribution product", Xi Tong Gong Cheng Yu Dian Zi Ji Shu/Systems Engineering and Electronics, vol. 42, no. 9, pp. 2140-2148, 2020.

[2] X. Zhou, "Research on group decision support system of product design evaluation based on Network", Northwest University of Technology, 2007.

[3] S. Lou, Y. Feng, Z. Li, H. Zheng, J. Tan, “An integrated decision-making method for product design scheme evaluation based on cloud model and EEG data", Advanced Engineering Informatics, vol. 43, pp. 101028, 2020.

[4] X. H. Chen, Theory and application of decision support system, Tsinghua university press. 2000.

[5] M. Z. Wang, P. Yuan, "Comparison of the outstanding design culture of Northern Europe and Japan and the construction of the Chinese modern design aesthetics", Packaging Engineering, vol. 26, no. 6, pp. 183-185, 2005.

[6] X. Wang, B. Zhang, M. Huang, F. Chang, Z. Zhou, "Improved LDA Model for Credibility Evaluation of Online Product Reviews", IEICE TRANSACTIONS on Information and Systems, vol. 102, no. 11, pp. 2148-2158.

[7] H. Li, B. S. Yang, "Model evaluation of particle breakage facilitated process intensification for Mixed-SuspensionMixed-Product-Removal (MSMPR) crystallization”, Chemical Engineering Science, vol. 207, pp. 1175-1186.

[8] H. Tan, J. Zhao, W. Wang, J. Zhang, "Thinking model and application in product form design", Journal of Mechanical Engineering, vol. 2006, no. B05, pp. 98-102, 2006.

[9] L. P. Hao, C. Liang, Q. Tan, C. Li, "Study on method and application in construction project bidding evaluation", Journal of Tianjin University (Science and Technology), vol. 34, no. 4, pp. 515-520, 2001.

[10]D. Konsta, I. Binietoglou, A. Gkikas, S. Solomos, E. Marinou, E., Proestakis, V. Amiridis, "Evaluation of the BSC-DREAM8b regional dust model using the 3D LIVAS-CALIPSO product," Atmospheric Environment, vol. 195, pp. 46-62, 2018.

[11] Y. J. Wang, X. Y. Meng, "Research on the Method of Multilevel Fuzzy Evaluation of the Green Produ cts," China Mechanical Engineering, vol. 11, no. 9, pp. 10161019, 2000.

[12] A. Marin, S. Rossi, D. Burato, A. Sina, M. Sottana, “A product-form model for the performance evaluation of a bandwidth allocation strategy in WSNs," ACM Transactions on Modeling and Computer Simulation (TOMACS), vol. 28, no. 2, pp. 1-23.

[13] A. Zhou, J. Su, S. Yan, J. Ouyang, C. Shi, B. Yuwang, "Nonlinear information dynamics model of synthetic evaluation on product form aesthetic", Journal of Mechanical Engineering, vol. 54, no. 15, pp. 150-159, 2018.

[14] R. Q. Yang, "Design and operation of engineering system," Shanghai Jiaotong University Press. 2004.

[15] D. Acevedo, Y. Yang, D. J. Warnke, Z. K. Nagy, "Modelbased evaluation of direct nucleation control approaches for the continuous cooling crystallization of paracetamol in a mixed suspension mixed product removal system", Crystal Growth \& Design, vol. 17, no. 10, pp. 5377-5383, 2017.

[16] T. Saouabe, E. M. El Khalki, M. E. M. Saidi, A. Najmi, A. Hadri, S. Rachidi, Y. Tramblay, "Evaluation of the GPMIMERG precipitation product for flood modeling in a semiarid mountainous basin in Morocco," Water, vol. 12, no. 9, pp. 2516, 2020.

[17] M. Lin, P. Papadopoulos, "Formula-one 3D Vehicle Aerodynamic Modeling, Geometric Idealization and Mesh Refinement Strategies", WSEAS Transactions on Fluid Mechanics, vol. 14, Art. \#6, pp. 40-61, 2019.

[18] R. Montemanni, L. M. Gambardella, F. Cellina, F. Cartolano, P. Cossu, A. Rizzoli, "Current and Future Challenges for Urban Mobility", WSEAS Transactions on Computers, vol 18, Art. \#18, pp. 136-140, 2019.

[19] R. Singh, P. Tandon, "User values based evaluation model to assess product universality", International Journal of Industrial Ergonomics, vol. 55, pp. 46-59, 2016.

[20] O. Aydemir, "Odor and subject identification using electroencephalography reaction to olfactory", Traitement du Signal, vol. 37, no. 5, pp. 799-805, 2020.

[21]M. S. Bascil, "Jaw-operated human computer interface based on EEG signals via artificial neural networks", Revue d'Intelligence Artificielle, vol. 34, no. 1, pp. 21-27, 2020.

[22] H. Akbari, M. T. Sadiq, M. Payan, S. S. Esmaili, H. Baghri, Bagheri, H. "Depression detection based on geometrical features extracted from SODP shape of EEG signals and binary PSO”, Traitement du Signal, vol. 38, no. 1, pp. 1326, 2021.

[23] G. A. Garces, E. Bonjour, A. Rakotondranaivo, "New product acceptability evaluation and improvement model with knowledge reuse", IFAC-Papers Online, vol. 49, no. 12, pp. 1104-1109, 2016.

[24] D. Ding, L. Zhang, J. L. Liu, F. Chen, X. R. Si, “Design and implementation of a comprehensive evaluation model for shallow groundwater based on matter element extension", International Journal of Design \& Nature and Ecodynamics, vol. 15, no. 5, pp. 685-689, 2020.

[25]Z. H. Yang, "Analysis of the impacts of open residential communities on road traffic based on AHP and fuzzy theory", Ingénierie des Systèmes d'Information, vol. 25, no. 2, pp. 183-190, 2020.

[26] X. J. Zhu, Z. Jiang, X. B. Zhao, M. J. Zhang, X. F. Chen, "A novel fuzzy fusion algorithm of multi-sensor data and its application in coalmine gas monitoring", Instrumentation Mesure Métrologie, vol. 18, no. 6, pp. 577$582,2019$.

[27]Z. X. Sun, "The Application of Fuzzy Mathematics Theory in the Shape Design of the Products", Development \& Innovation of Machinery \& Electrical Products, vol. 21, no. 4, pp. 79-80, 93, 2008.

[28] W. R. Jing, "Application of Fuzzy Mathematics in Engineering Design”, China Mine Engineering, vol. 34, no. 2, pp. 35-37, 2005.

Wenhui Hou was born in Tangshan County,Hebei Province in 1982 . She received Bachelor of Engineering Degree in 
industrial design in 2006 and master degree in Mechanical Engineering in 2009.

Since 2012, she has been an Assistant Professor with the Mechanical Engineering Department, Tangshan Polytechnic College. She is the author of three books, her research interests include product design and research, digital design, and reverse engineering, etc. She is a member of the expert 1 for the World Skills Contest. Email: wenhui9909@163.com

Caiwen Niu. She was born in 1982 in Qingyuan County, Liaoning Province. She received master degree from Northeast Electric Power University in 2007. Now she is an associate professor in the Department of Automation Engineering, Tangshan Polytechnic College. Her research interests are mechatronics technology, automatic control technology, etc. Email: niucaiwen0305@126.com

\section{Author Contributions:}

Wenhui Hou conducted research conception, design and data analysis.

Caiwen Niu conducted data collection and Manuscript Preparation.

\section{Creative Commons Attribution License 4.0 (Attribution 4.0 International, CC BY 4.0)}

This article is published under the terms of the Creative Commons Attribution License 4.0

https://creativecommons.org/licenses/by/4.0/deed.en_US 